\title{
QUELQUES CAS D'ASPERGILLOSE AVIAIRE OBSERVES AU PARC ZOOLOGIQUE DE PARIS : LE PARASITE ET L'HOTE
}

\author{
Par Henri SAEZ
}

Tous les ans les Oiseaux de la Collection du Parc zoologique de Paris paient leur tribut à une mycose, l'Aspergillose, qui touche avec prédilection l'appareil respiratoire et peut parfois se généraliser. Chez 166 sujets autopsiés de novembre 1958 à février 1961 inclus, 35 avaient des lésions mycosiques. Mais ces 35 Oiseaux ne sont pas tous morts d'Aspergillose car, dans quelques cas, les lésions pulmonaires discrètes ou les implantations précaires d'un Aspergillus sur les sacs aériens étaient de véritables découvertes d'autopsie. Nous avons cependant retenu tous les cas, même ceux où le champignon semblait en voie d'exclusion de l'organisme, pour connaître les parasites et les hòtes susceptibles de les héberger.

Outre l'examen direct et quelquefois des coupes histologiques, des cultures sont faites systématiquement avec du matériel prélevé au niveau même des lésions. Les primo-cultures sont obtenues en boìtes de Pétri, coulées avec du milieu de Sabouraud glucosé à $2 \%$, dont les unes sont gardées à la température du laboratoire et les autres portées à l'étuve à $37^{\circ}$. Plusieurs milieux (milieu de Czapek à 3 et $20 \%$ de saccharose, milieu à l'amidon de maïs à $1 \%$, fragments de carotte ou de pomme de terre en tubes de Roux, Maltea-Moser à 1 et $2 \%$ ) ont été utilisés pour l'étude des souches (sporulation, appareil conidien, pigmentation, production de périthèces...). Pour l'identification, nous nous sommes reportés à l'ouvrage de Thом and Raper (1).

\section{Le Parasite}

Les lésions mycosiques constatées chez 35 Oiseaux étaient dues :

- 32 fois à l'Aspergillus fumigatus Fresenius,

- 1 fois à l'Aspergillus amstelodami (Mang.) Thom et Raper,

- 1 fois à l'Aspergillus terreus Thom et à un Penicillium sp.,

- 1 fois à l'Aspergillus flavus Link. 

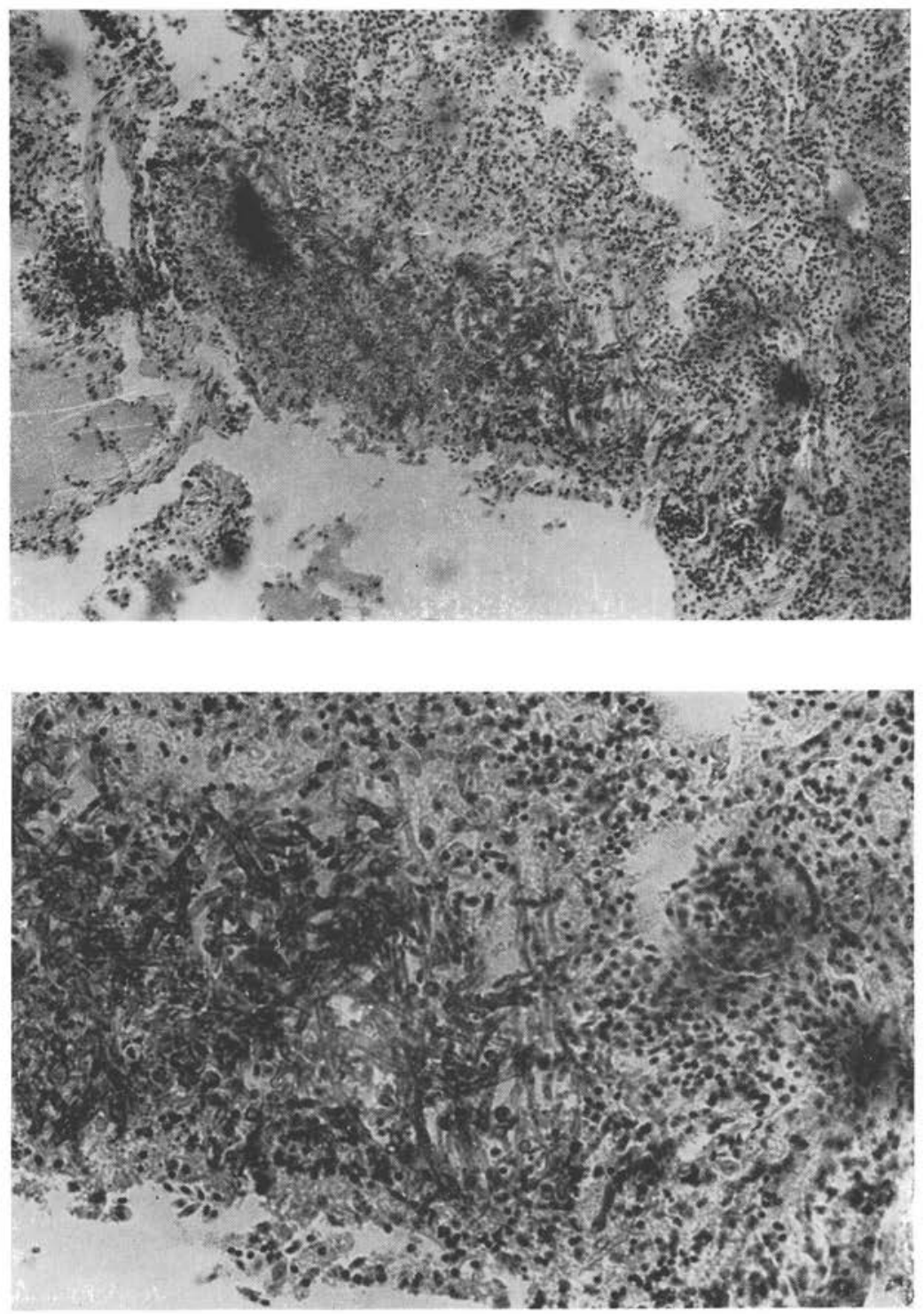

Fıg. 1. - Aspergillose généralisée chez un Manchot royal (Aptenodytes patagonica Miller). Filaments mycéliens dans une coupe de rein.

Fiti. 2. - Détail de la figure précédente 
L'Aspergillus fumigatus Fresenius est en cause dans tous les cas graves et mortels d'Aspergillose et dans la grande majorité des lésions plus discrètes découvertes à l'autopsie. Cependant, il convient de signaler les faits suivants :

- l'on peut rencontrer l'Aspergillus fumigatus Fresenius dans le poumon de sujets exempts de mycose, aussi bien d'ailleurs chez des Oiseaux que chez des Mammifères (2);

- dans des cas d'Aspergillose de l'appareil respiratoire provoqués par l'Aspergillus fumigatus Fresenius, il nous a été possible d'isoler dans le poumon (en zones saines), dans la trachée ou les sacs aériens, des Aspergillus d'espèces différentes.

Des spores de champignons très divers sont introduites par la respiration. Ces hôtes occasionnels, fréquents, ne doivent pas être confondus avec le parasite. Parmi les seuls Aspergillus le plus souvent rencontrés, citons, en dehors de l'Aspergillus fumigatus Fresenius, l'A spergillus flavus Link, l'A spergillus nidulans (Eidam) Wint. et l'Aspergillus candidus (une souche de cette dernière espèce, se développant à $37^{\circ}$, a fréquemment été isolée au Laboratoire dans des prélèvements d'origine animale: poumon, pharynx, contenu intestinal, etc.).

L'Aspergillus amstelodami (Mang.) Thom et Raper a été identifié au niveau de granulations de la grosseur d'une tête d'épingle, au nombre d'une dizaine, parsemées sur les sacs aériens d'un Pélican à bec tacheté (Pelecanus philippensis Gm.). Dans ces granulations calcifiées, difficiles à écraser entre deux lames, nous avons trouvé des filaments, souvent altérés, de mycelium stérile. Des périthèces jaunes se sont produits abondamment sur la plupart des milieux. Les asques, à maturité, contenaient des ascospores lenticulaires à subsphériques, épineuses, à sillon équatorial bien marqué, sans membranules partant de ses crêtes.

L'Aspergillus terreus Thom ainsi qu'un Penicillium sp. ont été isolés à partir d'un nodule kystique de 4-5 $\mathrm{mm}$. de diamètre siégeant dans un diverticule du sac aérien droit d'un Cygne noir (Chenopsis atrata Latham). Dans ce nodule, il y avait des filaments mycéliens et quelques rares têtes aspergillaires portant deux rangées de phialides.

Chez le Pélican à bec tacheté (Pelecanus philippensis $\mathrm{Gm}$.) comme chez le Cygne noir (Chenopsis atrata Latham), les implantations dans l'organisme sont précaires et les champignons semblent en voie d'exclusion. Aussi ne peut-on pas considérer, pour l'instant, l'Aspergillus amstelodami (Mang.) Thom et Raper et l'Aspergillus terreus (et le Penicillium sp.) comme des espèces pathogènes pour 
les Oiseaux du Parc, comparables à l'Aspergillus fumigatus Fresenius.

L'Aspergillus flavus Link formait une véritable petite colonie in vivo à la face interne du sternum d'un Flamant rouge (Phoenicopterus ruber L.). Une petite touffe de filaments blancs ou vert-

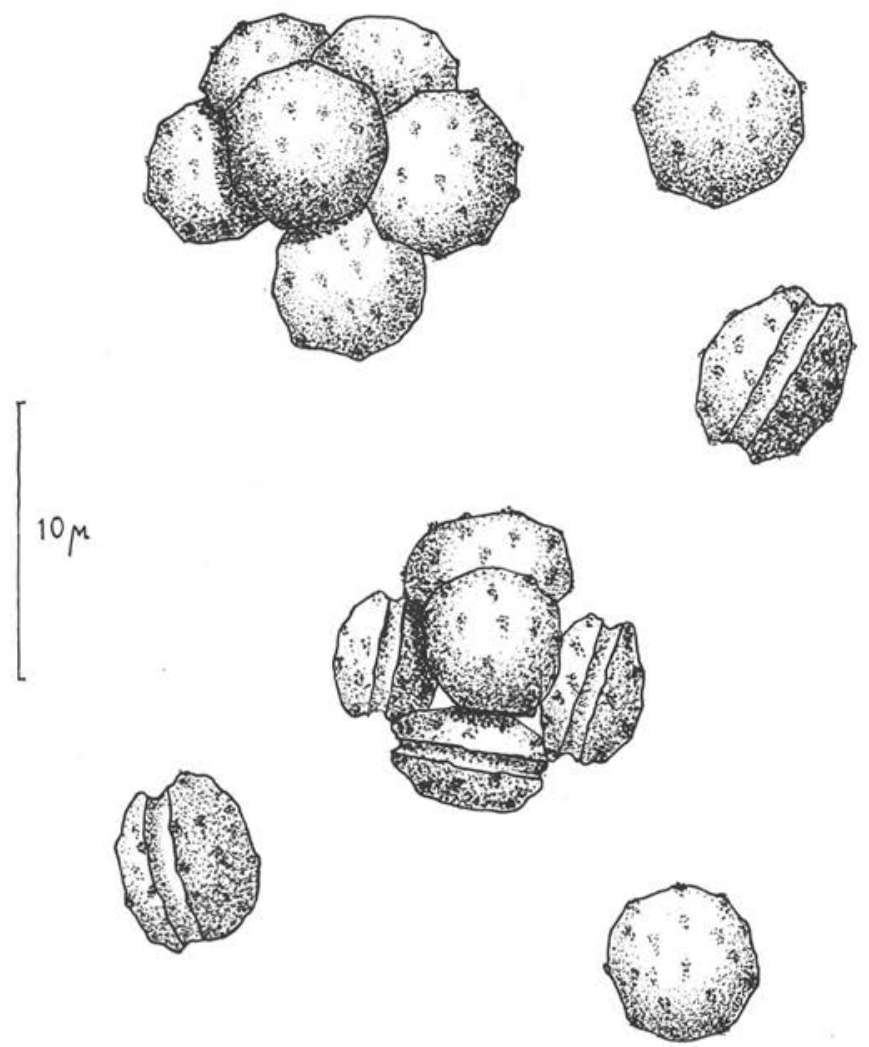

Fig. 3. - Ascospores d'Aspergillus amstelodami (Mangin) Thom et Church (culture de deux mois sur milieu de Czapek). Dessin à la chambre claire

jaunâtre avait pris naissance sur une surface de $1 \mathrm{~cm}$. de diamètre environ sur la paroi thoracique médiane, un peu au-dessus du cœur. Les cultures ont permis l'identification d'un Aspergillus flavus Link que l'on pouvait suspecter à l'examen de cette colonie encore jeune : conidiophores finement ponctués, beaucoup plus de têtes aspergillaires à une seule rangée qu'à deux rangées de phialides, conidies peu pigmentées, rondes, à peine échinulées. 


\section{L'Hôte}

L'hôte se recrute principalement parmi les trois familles suivantes :

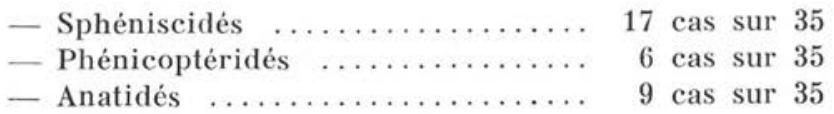

\section{Les Sphéniscidés.}

Des Manchots de Humboldt (Spheniscus humboldti Meyen) et des Manchots du Cap (Spheniscus demersus L.) mènent une vie prospère en captivité. Tandis que nous n'avons jamais constaté un seul cas d'Aspergillose chez ces animaux, dont plusieurs sont nés au Parc, cette mycose fait par contre de grands ravages dans des lots d'animaux importés des Iles Kerguelen et comprenant des Manchots papous [Pygoscelis papua (Forster)], des Gorfous dorés [Eudyptes chrysolophus (Brandt)] et des Manchots royaux (Aptenodytes patagonica Miller). Les Manchots papous et les Gorfous dorés sont les moins résistants. Quant aux Manchots royaux, il existe toujours une petite colonie de ces animaux; dans un lot de sept à huit sujets importés, un ou deux en moyenne résistent à l'Aspergillose. Ceux-ci s'acclimatent bien et, par la suite, les cas mortels dus à cette mycose ne sont pas plus fréquents chez eux que chez les autres espèces d'Oiseaux du Parc.

\section{Les Phénicoptéridés.}

Il existe des cas isolés chez les Flamants roses (Phoenicopterus antiquorum Tem.) et les Flamants nains [Phoeniconaias minor (Geoffroy)]. Chez les Flamants rouges (Phoenicopterus ruber L.), la maladie frappe souvent, comme précédemment, des animaux récemment arrivés au Parc.

\section{Les Anatidés.}

C'est dans cette famille que se recrutent la plupart des cas autochtones du Parc : oiseaux bien acclimatés, vivant depuis longtemps, ou même nés en captivité. Il y a une grande diversité d'espèces sensibles à l'Aspergillose, mais il faut dire aussi que cette famille est l'une des mieux représentées au Parc.

D’une façon générale, chez tous les Ciseaux, il existe des espèces plus réceptives que d'autres (remarquons le grand nombre d'Oiseaux aquatiques), mais les conditions favorisantes ont leur importance. Les fatigues d'un long voyage, le jeûne que ce voyage entraîne quelquefois, le dépaysement, les périodes critiques de la vie (mue, croissance), favorisent le développement d'une Aspergillose. 
Nous avons pu observer quelques lots d'animaux provenant des Iles Kerguelen. Le sujet malade cherche à s'isoler et à s'immobiliser dans un coin tranquille du box. L'issue fatale est annoncée, deux ou trois jours avant, rarement davantage, par une très forte dyspnée: le bec ouvert, la cage thoracique déprimée, les muscles contractés, l'animal lutte surtout pour rejeter l'air. Chaque effort est suivi d'une courte pause. Nous n'avons pas constaté de dyspnée chez les Flamants rouges d'un lot que nous avions suivi depuis son arrivée, et pourtant certains sont morts avec des lésions massives de tout le parenchyme pulmonaire.

Si l'Aspergillose touche primitivement l'appareil respiratoire avec prédilection, les formes extra-pulmonaires et localisées aux sacs aériens ne sont pas rares à la période de début. Les sacs aériens sont en contact avec la trachée, chez les Oiseaux, et peuvent recevoir directement de l'air, donc des spores de champignons pathogènes. Les lésions discrètes consistent en granulations sur les sacs aériens ou petits nodules infiltrant le parenchyme pulmonaire. Le parasite peut se développer en larges plaques de mycélium fertile (les conidies ensemençant les régions alentour), qui tapissent la paroi des sacs aériens, la surface des poumons et forment des manchons autour de la trachée et des gros vaisseaux à la sortie du cœur. A l'intérieur des bronches et dans la lumière de la trachée, on peut parfois observer un épais feutrage mycélien. Le parasite s'infiltre parfois dans la cavité abdominale et on assiste à une généralisation de l'Aspergillose.

L'Aspergillome n'est pas une rareté chez les Oiseaux. Il se voit moins fréquemment chez les Sphéniscidés que chez les Phénicoptéridés ou les Anatidés.

\section{RÉSUMÉ}

Chez 35 Oiseaux présentant des lésions mycosiques à l'autopsie, 32 fois nous avons isolé l'Aspergillus fumigatus Fresenius. Cette espèce est, sinon la seule, du moins la plus fréquente et la plus pathogène pour les Oiseaux vivant en captivité au Parc zoologique. Les sujets les plus réceptifs à l'Aspergillose sont les Manchots royaux (Aptenodytes patagonica Miller), les Manchots papous [Pygoscelis papua (Forster)], les Gorfous dorés [Eudyptes chrysolophus (Brandt)], les Flamants rouges [Phoenicopterus ruber (L.)] et diverses espèces de Canards. Mais, outre la sensibilité de l'hôte, qui a souvent l'occasion d'être en contact avec des spores de champignons pathogènes, des conditions sont favorisantes au développement d'une Aspergillose telles : les fatigues d'un long voyage, la non-acclimatation, des périodes critiques de la vie (mue, croissance) et, d'une façon générale, toute baisse de la résistance d'un sujet. 
TABLEAU I

Répartition des cas d'Aspergilose chez les différentes espèces animales

Famille des STRUTHIONIDES

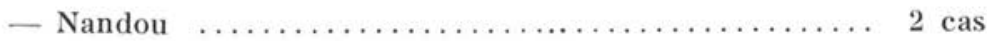
Rhea americana (L.)

Famille des SPHENISCIDES :

- Manchot royal ....................... 8 cas

$\begin{aligned} & \text { Aptenodytes patagonica Miller } \\ \text { - Manchot papou } . \ldots \ldots \ldots \ldots \ldots \ldots \ldots \ldots \ldots \ldots \ldots \ldots \ldots & 4 \text { cas }\end{aligned}$ Pygoscelis papua (Forster)

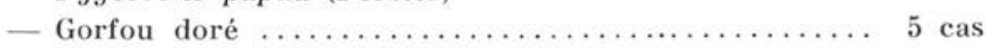
Eudyptes chrysolophus (Brandt)

Famille des PÉLECANIDÉS

- Pélican à bec tacheté $\ldots \ldots \ldots \ldots \ldots \ldots \ldots \ldots \ldots \ldots \ldots \ldots$ cas Pelecanus philippensis Gm.

Famille des PHENICOPTERIDES :

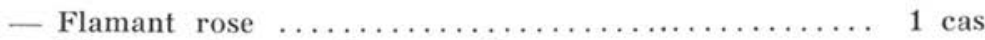
Phœnicopterus antiquorum Tem.

- Flamant rouge $\ldots \ldots \ldots \ldots \ldots \ldots \ldots \ldots \ldots \ldots \ldots \ldots \ldots \ldots \ldots$ cas Phœnicopterus ruber $\mathrm{L}$.

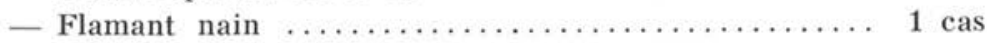

Famille des ANATIDES :

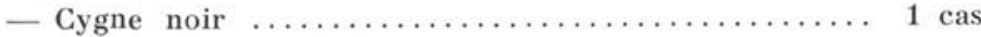
Chenopsis atrata Latham

- Oie à tête barrée $\ldots \ldots \ldots \ldots \ldots \ldots \ldots \ldots \ldots \ldots \ldots \ldots \ldots \ldots \ldots \ldots \ldots$ cas Eulabeia indica (Latham)

- Bernache à cou roux $\ldots \ldots \ldots \ldots \ldots \ldots \ldots \ldots \ldots \ldots$ cas Branta ruficollis (Pallas)

- Canard carolin ......................... 1 cas Aix sponsa L.

- Canard à faucille .......................... 1 cas Anas falcata Georgi

- Canard mandarin ........................ 1 cas Dendronessa galericulata L.

- Canard siffleur .......................... 1 cas Mareca penelope (L.)

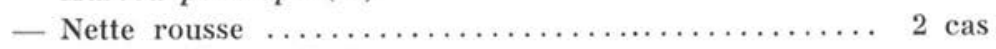
Netta rufina (Pallas) 
TABLEAU II

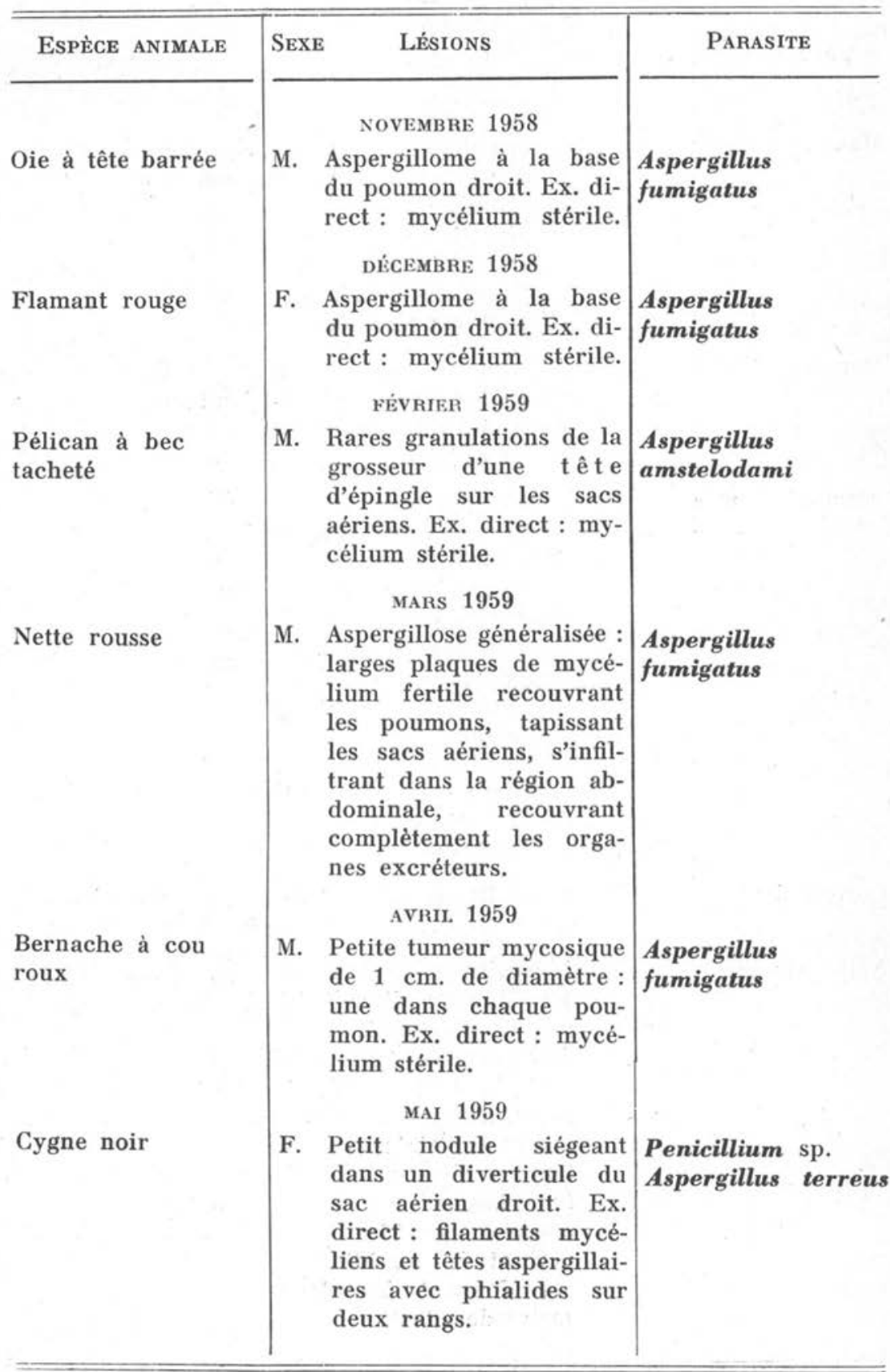


TABLEAU II (2)

Espèce animale $\mid$\begin{tabular}{ll} 
Sexe & Lésions \\
\hline
\end{tabular}

Manchot royal

Manchot royal

Flamant rouge

Gorfou doré

Nette rousse

M. Aspergillose extra-pulmonaire: une plaque de mycélium fertile de 2 $\mathrm{cm}$. de diamètre et quelques rares lésions lenticulaires (mycélium stérile) sur les sacs aériens.

M. Lésions discrètes d'aspergillose extra-pulmonaire (parois thoraciques et sacs aériens).

F. Tout le parenchyme pulmonaire infarci de nodules de 1-2 mm. à plus de $1 \mathrm{~cm}$. de diamètre et tapis verdâtre de mycélium fertile recouvrant les poumons en surface, les gros vaisseaux à la sortie du cœur, les sacs aériens. Ex. direct des nodules : appareils conidiens typiques du groupe fumigatus.

F. Aspergillose généralisée.

Aspergillus

fumigatus

Parasite

Aspergillus

fumigatus

Aspergillus

fumigatus

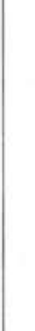

Aspergillus

fumigatus

F. Aspergillose généralisée. Aspergillus

Ex. direct : dimorphis- fumigatus

me de l'appareil conidien : têtes aspergillaires typiques du groupe fumigatus, d'autres fortement massuées, étirées, rappelant les têtes d'Aspergillus clavatus (un même filament mycélien supportant des conidiophores donnant les deux sortes de têtes). 
TABLEAU II (3)

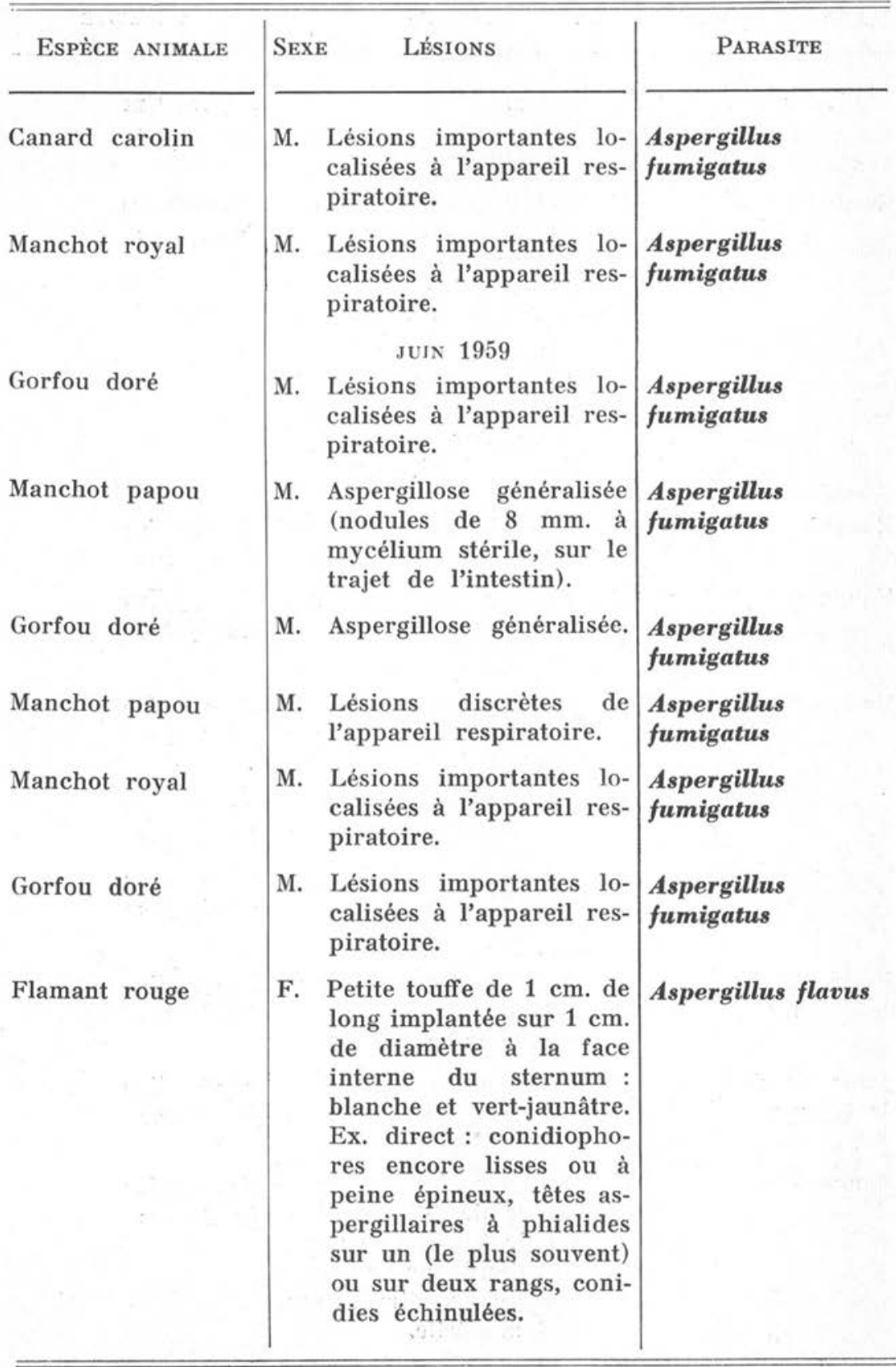


TABLEAU II (4)

\begin{tabular}{|c|c|c|c|}
\hline ESPĖCE ANIMALE & SEx & LÉSIONS & Parasite \\
\hline Gorfou doré & & $\begin{array}{l}\text { Lésions importantes lo- } \\
\text { calisées à l'appareil res- } \\
\text { piratoire. }\end{array}$ & $\begin{array}{l}\text { Aspergillus } \\
\text { fumigatus }\end{array}$ \\
\hline Canard à faucille & & $\begin{array}{l}\text { Aspergillose extra-pul- } \\
\text { monaire: plaques na- } \\
\text { crées sur les gros vais- } \\
\text { seaux à la sortie du } \\
\text { cour et sur les sacs } \\
\text { aériens. Plaques ligneu- } \\
\text { ses à la coupe. Ex. di- } \\
\text { rect : compact mycélien, } \\
\text { rares têtes aspergillaires } \\
\text { de type fumigatus. }\end{array}$ & $\begin{array}{l}\text { Aspergillus } \\
\text { fumigatus }\end{array}$ \\
\hline Manchot papou & & Aspergillose généralisée. & $\begin{array}{l}\text { Aspergillus } \\
\text { fumigatus }\end{array}$ \\
\hline Manchot royal & & $\begin{array}{l}\text { Lésions localisées à l'ap- } \\
\text { pareil respiratoire. } \\
\text { jUILLET } 1959\end{array}$ & $\begin{array}{l}\text { Aspergillus } \\
\text { fumigatus }\end{array}$ \\
\hline Manchot papou & & $\begin{array}{l}\text { Lésions importantes lo- } \\
\text { calisées à l'appareil res- } \\
\text { piratoire. }\end{array}$ & $\begin{array}{l}\text { Aspergillus } \\
\text { fumigatus }\end{array}$ \\
\hline Canard mandarin & & Aspergillose généralisée. & $\begin{array}{l}\text { Aspergillus } \\
\text { fumigatus }\end{array}$ \\
\hline Manchot royal & & $\begin{array}{l}\text { Aspergillose généralisée. } \\
\text { SEPTEMBRE } 1959\end{array}$ & $\begin{array}{l}\text { Aspergillus } \\
\text { fumigatus }\end{array}$ \\
\hline $\begin{array}{l}\text { Jeune Nandou } \\
\text { de } 8 \text { jours }\end{array}$ & & $\begin{array}{l}\text { Rares petits nodules de } \\
1-2 \text { mm. dans le paren- } \\
\text { chyme pulmonaire. }\end{array}$ & $\begin{array}{l}\text { Aspergillus } \\
\text { fumigatus }\end{array}$ \\
\hline $\begin{array}{l}\text { Jeune Nandou } \\
\text { de } 8 \text { jours }\end{array}$ & & $\begin{array}{l}\text { Rares petits nodules de } \\
\text { 1-2 mm. dans le paren- } \\
\text { chyme pulmonaire. }\end{array}$ & $\begin{array}{l}\text { Aspergillus } \\
\text { fumigatus }\end{array}$ \\
\hline Manchot royal & & $\begin{array}{l}\text { Quelques tumeurs myco- } \\
\text { siques de } 1-2 \mathrm{~cm} \text {. dans } \\
\text { les deux poumons; gra- } \\
\text { nulations sur les sacs } \\
\text { aériens. Ex. direct : my- } \\
\text { célium stérile. }\end{array}$ & $\begin{array}{l}\text { Aspergillus } \\
\text { fumigatus }\end{array}$ \\
\hline
\end{tabular}


TABLEAU II (5)

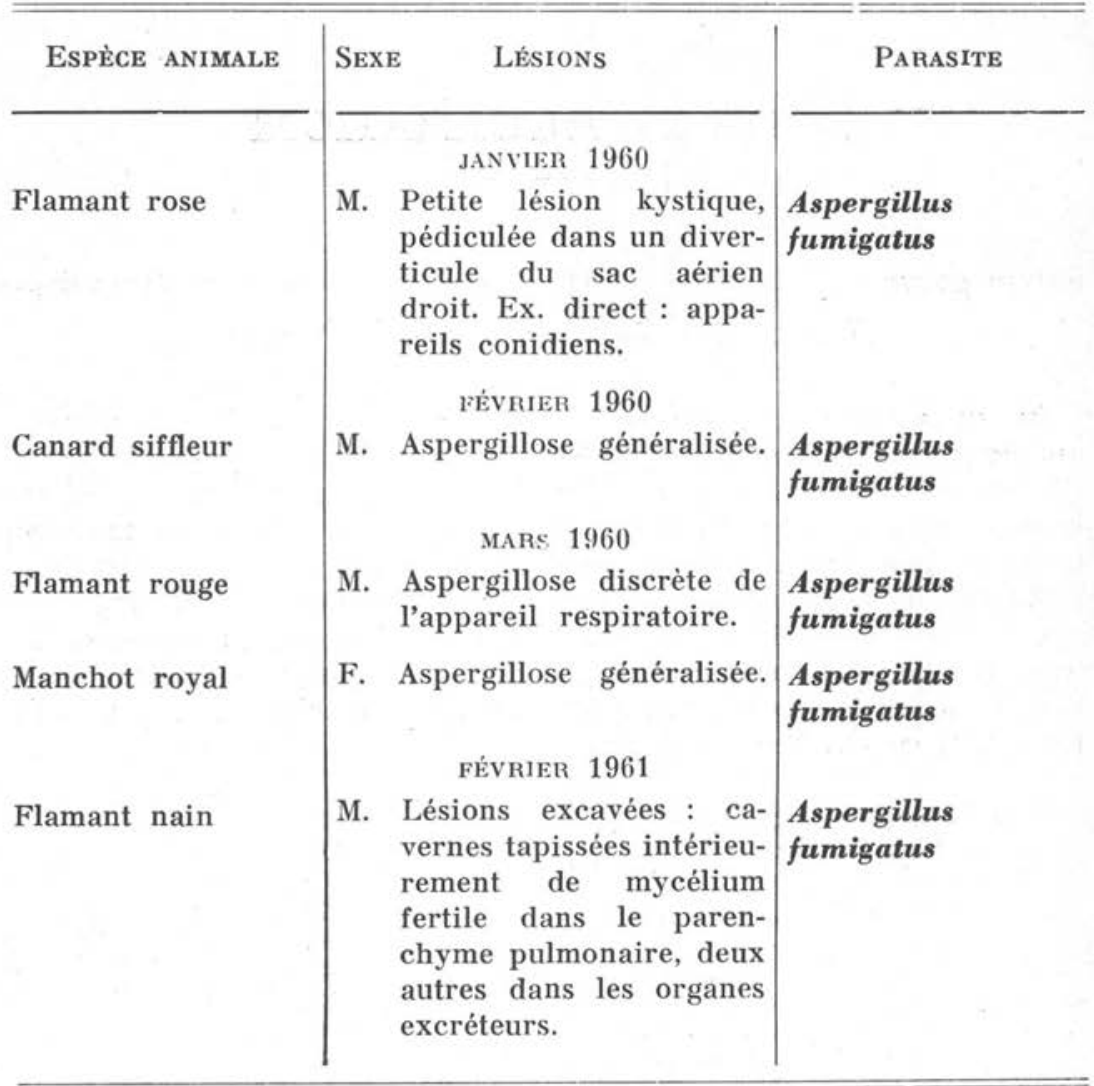

\section{BiBLIOGRAPHIE}

1. Thом (Ch.) et Raper (K. B.), 1945. - A manual of the Aspergilli, 1 vol., Baltimore, The Williams and Wilkins company.

2. SAEZ (H.), 1960. - Champignons isolés du poumon de quelques Mammifères sauvages morts en captivité. Parassitologia (Roma), vol. II, $\mathrm{n}^{\circ} 3$, pp. 353-358.

(Muséum National d'Histoire Naturelle

Parc Zoologique de Paris) 